\title{
Normalizing Rain Gauge Network Biases with Calibrated Radar Rainfall Estimates
}

\author{
Gary D. Martens and James T. Smullen
}

The identification and adjustment of precipitation time series data for nonclimatic changes in recording bias among rain gauges can be instrumental in controlling uncertainty in hydrologic models. Hydrologic models depend upon the reliability of precipitation and flow monitoring data sets used for calibration and simulation. Consistent precipitation and flow monitoring measurements clearly can be important when attempting to characterize rainfall runoff relationships over time. Hydrologic models require rain gauge networks to represent the spatial distribution of precipitation across a drainage basin and benefit from the normalization of relative rain gauge biases across the network.

Calibration of large urban sewer system models, using a moderately-dense basin-wide rain gauge network and continuous flow monitoring data, is improved by creating continuous homogeneous rainfall records with normalized spatial biases.

Double-mass regression and cumulative residual time series analysis techniques are used to evaluate and adjust historical rain gauge network data to correct for non-homogeneity of individual rainfall records and to normalize spatial bias across the network. Homogeneity of rainfall time series data is evaluated and adjusted by comparison to the rain gauge network mean over a 13-year period of record. Spatial bias across the network, then, is normalized by comparison to continuous calibrated radar rainfall estimates obtained over a 15-month period. Cumulative residual time series analysis techniques also are applied to evaluate the homogeneity of flow monitoring data used in model calibration. The benefits of normalizing the rain gauge network biases to model calibration are illustrated by comparing model results using gauge data with and without bias correction.

Martens, G. and J. Smullen. 2005. "Normalizing Rain Gauge Network Biases with Calibrated Radar Rainfall Estimates." Journal of Water Management Modeling R223-16. doi: 10.14796/JWMM.R223-16.

(C) CHI 2005 www.chijournal.org ISSN: 2292-6062 (Formerly in Effective Modeling of Urban Water Systems. ISBN: 0-9736716-0-2) 


\subsection{Introduction}

\subsubsection{Homogeneity of Rain Gauge Station Records}

Hydrologic model calibration of lumped runoff parameter estimates depends on consistent rather than precise absolute precipitation and flow monitoring measurement over time. Homogeneity of a rain gauge record refers to the consistency of non-climatic bias in precipitation measurements at a gauge location over its period of record. Changes in the method of measurement, location of the gauge, or conditions immediately surrounding the gauge, can cause the readings to differ systematically from prior readings and are indications of a need for correction (Easterling et al 1995). Homogeneity adjustment of rain gauge data is performed to create a consistently scaled rainfall record at each gauge location.

Adjustment of gauge data to form homogeneous time series depends upon the ability to identify times when changes in measurement conditions may have occurred (Alexandersson 1986). Meta-data, a gauge history record documenting changes in equipment and site conditions, often is used as the primary means for identifying changes in rain gauge measurement conditions (Guttman 1998). Meta-data alone, however, is often insufficient for identifying non-homogeneity of gauge records. Major reported equipment or station location changes can have little if any effect on the gauge record, whereas seemingly minor adjustments and undocumented changes in site conditions may result in profound changes in the rainfall record as identified by analytic methods (Peterson et al 1998).

Time series analysis methods used for evaluating homogeneity and adjusting rain gauge records depend upon comparison of gauge data to a homogeneous reference time series. An appropriate reference time series is created by averaging measurements from several highly correlated nearby gauges (Guttman 1998, Peterson et al 1998). It is also found valuable to include gauges with short and incomplete data series in the reference value (Alexandersson 1986).

\subsubsection{Normalizing rain gauge network biases}

Representing precipitation spatially across a water or sewer-shed depends upon consistent recording among gauges in a rain gauge network. Once homogeneous rain gauge records are created, it is important that all gauges in the network be scaled to combine data for use in filling missing records. The 
goal is to develop a continuous rainfall record for each gauge location, and to determine spatial bias adjustment factors to consistently represent the spatial distribution of rainfall across the network.

Normalizing rain gauge network biases in this manner depends upon a reliable reference precipitation data set that represents spatial variation across the region with a uniform bias over a sufficiently long period of record. Calibrated radar rainfall estimates are used for this purpose.

\subsubsection{Case Study}

The Philadelphia Water Department (PWD), as part of the City of Philadelphia's combined sewer overflow (CSO) permit compliance program, developed system hydraulic models of its separate sanitary and combined sewer systems that contribute flows to each of its three water pollution control plants, draining nearly 140 square miles of the city. The City maintains a network of 24 tipping bucket rain gauges as part of this program. In addition, the City has obtained 18 months of largely continuous historical gauge calibrated radar rainfall estimates provided by NEXRAIN Corporation, in order to further refine calibration of its large complex hydraulic system models. A map of Philadelphia showing approximate locations of PWD rain gauges, radar rainfall grid, as well as, the combined and sanitary sewer service areas is presented in Figure 16.1. Flow monitoring data from temporary PWD combined sewer flow monitoring site TC06 were also used for this study. The ninety-seven-acre drainage basin for site TC06 is located in the region north of PWD rain gauge 22 as seen in Figure 16.1.

Comparison of long term rainfall accumulations at neighboring gauges revealed potentially significant systematic differences in non-climatic biases. Double mass and cumulative residual analyses of gauge station records against the gauge network mean value have further revealed non-homogeneity of station records due to changes in equipment operation or site conditions. Adjustment of rain gauge data, therefore, was applied to create a consistently scaled precipitation record at each gauge location. In addition to creating consistent (homogeneous) gauge records over time, it also is important to scale all the gauges consistently within the network to one another in order to combine data from different gauges, for use in filling missing records, and representing spatial variation.

The goals of this investigation are to develop procedures to evaluate and adjust the historic PWD rain gauge network record to produce homogeneous 
rain gauge records at each gauge location and to normalize rain gauge network biases using radar rainfall estimates.

A preliminary evaluation of the effects of applying these time series homogenization and spatial bias normalization procedures on stormwater runoff parameter estimates and on hydrologic model reliability is also presented. In addition, application of these techniques for use in evaluating the consistency of flow monitoring data is also demonstrated.

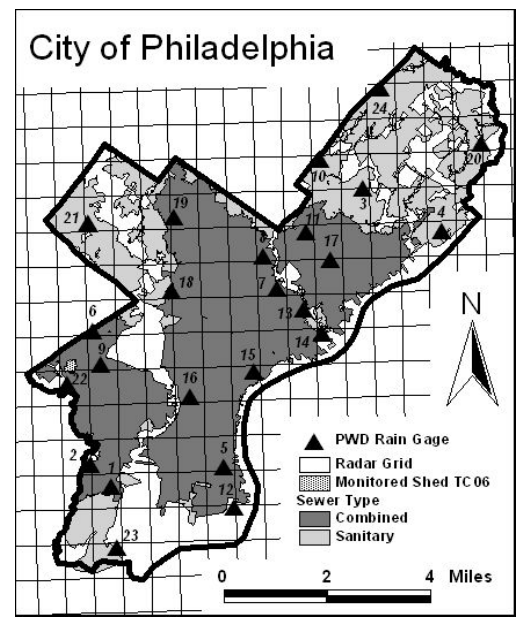

Figure 16.1 City of Philadelphia showing the approximate locations of PWD rain gauges, radar rainfall grid, as well as in city combined and sanitary sewer service areas.

\subsection{Homogeneity of PWD Rain Gauge Station Records}

This chapter describes the methods used to evaluate and adjust PWD rain gauge records to create continuous homogeneous rainfall records at each location in the network for use as input to hydrologic models.

\subsubsection{Data Set}

The PWD maintains a database of 15 -minute accumulated precipitation totals collected from its 24 tipping bucket rain gauge network for the period 1990 to 
the present. The uncorrected, 2.5-minute accumulated, 0.01 inch tip count, rain gauge data are subjected to preliminary quality assurance and quality control procedures. Identification and flagging of bad or missing data are performed for each rainfall event on a monthly basis by visual inspection of 15-minute accumulated data comparing measurements at nearby gauges and looking for patterns of obvious gauge failures, including plugged gauges and erratic tipping. Flagged data for each gauge subsequently are filled with data from the five nearest gauges using inverse distance squared weighting. Neighboring gauge data that are flagged are removed prior to weighting.

Daily rainfall volumes were totaled for each gauge. Daily gauge totals containing any filled data were removed from further analysis. A daily mean total was calculated for each gauge as the average of all daily gauge totals excluding the total at the gauge itself. The resulting dataset consisted of daily gauge totals, and daily mean totals (mean of all the other gauges) for each gauge.

\subsubsection{Double Mass Regression and Cumulative Residual Time Series Analysis}

Double mass regression and cumulative residual time series analysis methods were used for evaluating the homogeneity and adjusting PWD rain gauge records. These methods, like other reliable analytical methods of homogenizing rainfall time series, depend upon comparison of gauge data to a homogeneous reference series. A reference series can be created using the average of a collection of nearby gauges, or a homogeneous record at a single nearby gauge (Allen et al 1998). The rain gauge network mean value, calculated as described above, was selected as the reference series for homogenization of PWD rain gauge data.

Evaluating the homogeneity of PWD rain gauge records, and identifying dates when apparent changes in measurement conditions may have occurred, was performed using double mass and cumulative residual time series analysis techniques. A series of graphs was produced comparing gauge to mean daily rainfall totals for each gauge in the network. An example of the output generated is presented for PWD rain gauge 22 in Figures 16.2 and 16.3.

A double mass plot of gauge to mean cumulative daily rainfall totals was produced for each gauge. The slope of the linear regression line passing through the origin is referred to as the double mass regression slope, DMRS, as shown in Figure 16.2. Potential heterogeneities are identified by visual 
inspection of the double mass plot as seen by systematic departures from the trend line. These departures can be identified more easily by plotting the accumulated residual from the simple linear regression of gauge against mean daily rainfall totals over time (Craddock 1979) as shown in the cumulative residual plot in Figure 16.3.

Evaluation of potentially significant gauge record non-homogeneity was aided by the addition of an objective graphic analytic tool to the cumulative residual plot. An ellipse was drawn on the plot to contain the residual of a homogeneous time series for a given probability of the standard normal variate (Allen et al 1998, Henriques et al 1999). The 80\% probability level, commonly used by others according to Allen et al 1998, was chosen for this data evaluation program. Because the cumulative residual time series plot in Figure 16.3 is not contained within the ellipse, we reject at the $80 \%$ confidence level the hypothesis that the rainfall record at PWD rain gauge 22 is homogeneous with respect to the mean. The parametric equation defining the probability ellipse is given by (Allen et al 1998)

$$
\begin{aligned}
& x=\alpha \operatorname{Cos}(\theta)+\alpha \\
& y=\beta \operatorname{Sin}(\theta)
\end{aligned}
$$

with

$$
\begin{aligned}
& \alpha=n / 2 \\
& \beta=n Z_{p} S_{y, x} / \sqrt{(n-1)} \\
& S_{y, x}=S_{y} \sqrt{\left(1-r^{2}\right)}
\end{aligned}
$$

where:

$$
\begin{aligned}
n & =\text { the number of observations } \\
S_{y} & =\text { the sample standard deviation } \\
r & =\text { the Pearson correlation coefficient } \\
Z_{p} & =\text { the standard normal variate at } 80 \% \text { probability } \\
\theta & =\text { an angle in radians varying from } 0 \text { to } 2 \pi
\end{aligned}
$$

Cumulative residual analysis reveals even subtle change in gauge bias often undetected by routine inspection of precipitation data and gauge history records. Furthermore, subjective evaluation of the cumulative residual plot enables effective identification of the approximate dates abrupt changes in the relationship between the gauge and its neighbors occur (Craddock 1979). In 
this manner, a set of adjustment periods were determined that contain continuous, relatively homogeneous segments of each gauge record. Objective statistical methods have been used by others to identify significant break points in gauge record homogeneity (Peterson et. al. 1998). These methods have not been used here, however, like the subjective method of defining homogeneous periods used in this data homogenization program, they rely on comparative evaluation of time series data from a moderately dense and highly correlated gauge network.

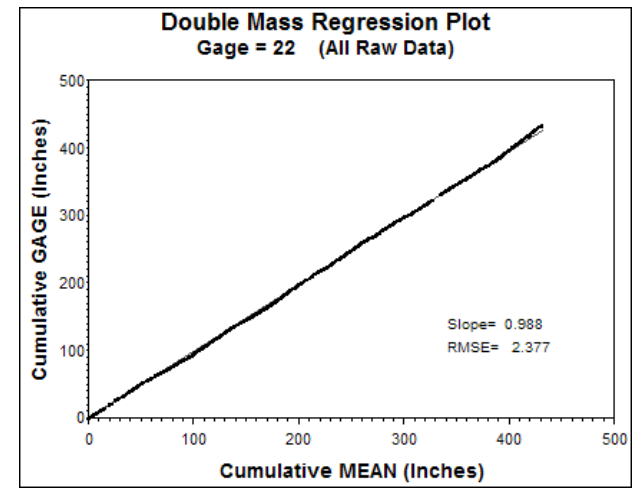

Figure 16.2 Double Mass Regression plot of cumulative daily rainfall at PWD rain gauge 22 against mean using all raw data for the 1990-2003 period of record.

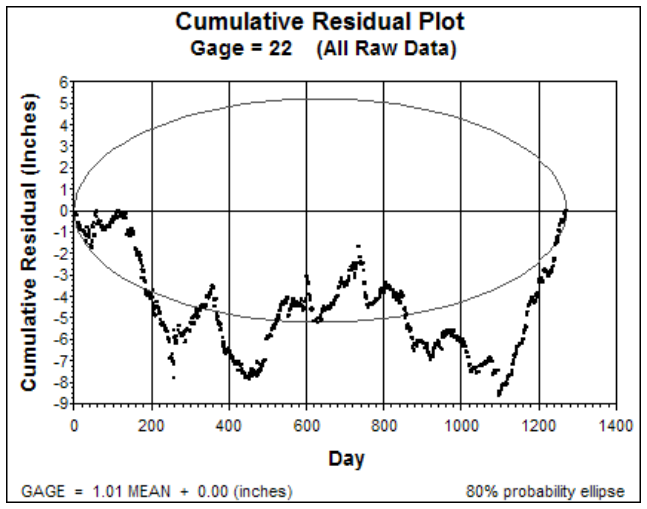

Figure 16.3 Cumulative residual time series plot from the linear regression of daily gauge against mean rainfall totals at PWD rain gauge 22 using all raw data for the period 1990-2003. 


\subsubsection{Adjusting Heterogeneous Gauge Records}

Once significant non-homogeneity of the gauge record is determined, and the limits of homogeneous adjustment periods have been identified, adjustment factors are computed for these periods to form a homogeneous data record.

Homogeneity adjustment factors are viewed as the ratio of the average gauge biases between a reference period and the period to be adjusted (Guttman 1998). Several methods of calculating adjustment factors were investigated. Each method employs a different form of expressing the average bias at the gauge relative to the mean. Three methods of estimating average biases for a gauge period that were considered are:

1. Average Daily Ratio of rain gauge value to the mean (high influence of small event outliers)

2. Double Mass Linear Regression Slope (most stable with respect to outliers)

3. Linear Regression Slope (high influence of large event outliers)

The double mass linear regression slope with y-intercept $=0$ was found to be the most stable with respect to outliers and was chosen for this study to determine homogeneity adjustment factors for selected periods of the rain gauge record.

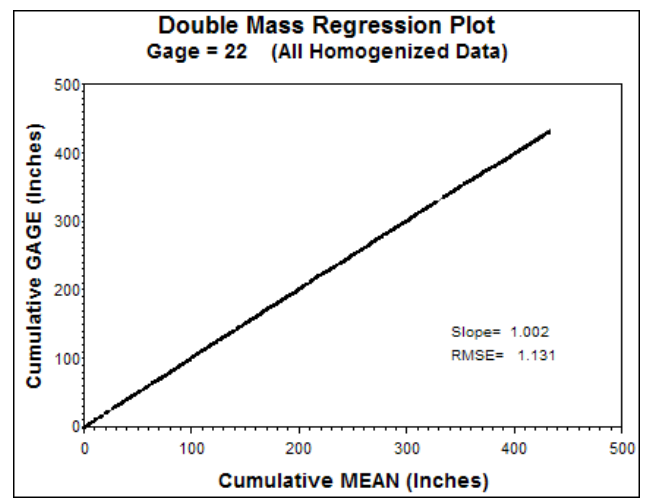

Figure 16.4 Double Mass Regression plot of cumulative daily rainfall at PWD rain gauge 22 against mean using homogenized data for the 19902003 period of record.

Rain gauge record homogeneity adjustment factors were calculated for each interval by dividing the double mass regression slope (DMRS) of the entire 
unadjusted data record (Figure 16.2) by the DMRS for the adjustment period. This calculation is performed for each adjustment period identified and all raw data within the adjustment periods then is multiplied by these factors to generate the corrected rain gauge record.

The results of homogeneity adjustment are presented for PWD rain gauge 22 with the double mass regression plot in Figure 16.4 and the cumulative residual plot in Figure 16.5. Comparison of these plots to those presented in Figures 16.2 and 16.3 for the raw data reveal a significant improvement in homogeneity of rain gauge bias relative to the network mean over the period of record for this gauge.

\subsection{Normalizing spatial biases in rain gauge data using calibrated radar rainfall estimates}

Once homogeneous gauge records are created, all gauges then are adjusted for consistent net systematic biases resulting from differences in gauge equipment, gauge site conditions, and previous homogeneity adjustments.

The second major goal of this investigation is to adjust all the gauges in the PWD network to the same average bias, so the gauge network more reliably represents spatial variation across the region. To achieve this goal, a reliable reference series is needed to represent spatial variation of rainfall across region with a uniform bias, over a sufficiently long and homogeneous period of record. Calibrated radar rainfall estimates are used for this purpose.

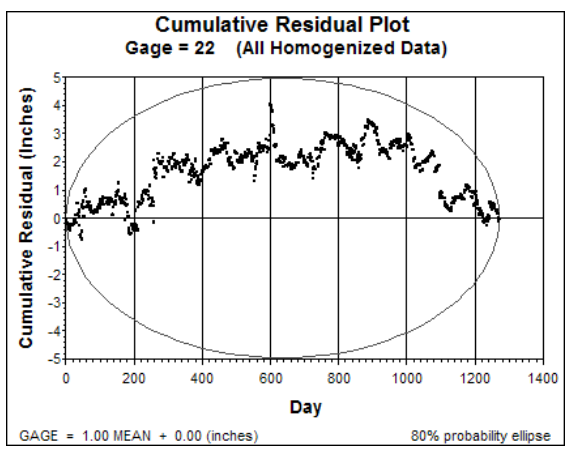

Figure 16.5 Time series plot of the cumulative residual from the linear regression of daily gauge against mean rainfall totals at PWD rain gauge 22 using homogenized data for the period 1990-2003. 


\subsubsection{Data Set}

Radar rainfall estimates provided by NEXRAIN Corporation were derived from a $2 \mathrm{~km}$ x $2 \mathrm{~km}$ National Weather Service level 3 radar mosaic product, corrected for ground clutter and other anomalies, and calibrated to the PWD 24 rain gauge network using a mean field bias adjustment. The 15-minute calibrated radar rainfall estimates for a 15-month period including two relatively recent intervals: October, 1999 through August, 2000 and March, 2002 through June, 2002 were used for this analysis. The radar rainfall estimates were calibrated to the PWD rain gauge network using a mean field bias adjustment method where the mean event accumulation for the radar pixels containing the PWD rain gauges is set equal to the mean event accumulation measured at the gauges. In this way the total volume of rainfall reported within the network is conserved, while the spatial variation represented by radar data is retained.

\subsubsection{Bias Adjustment Using Double Mass Regression}

Double mass regression analysis was used to correlate PWD rain gauge measurements to calibrated radar rainfall data. In this way, overall spatial bias adjustment factors are determined that best represent the spatial distribution of rainfall over the full period of record for each rain gauge in the network.

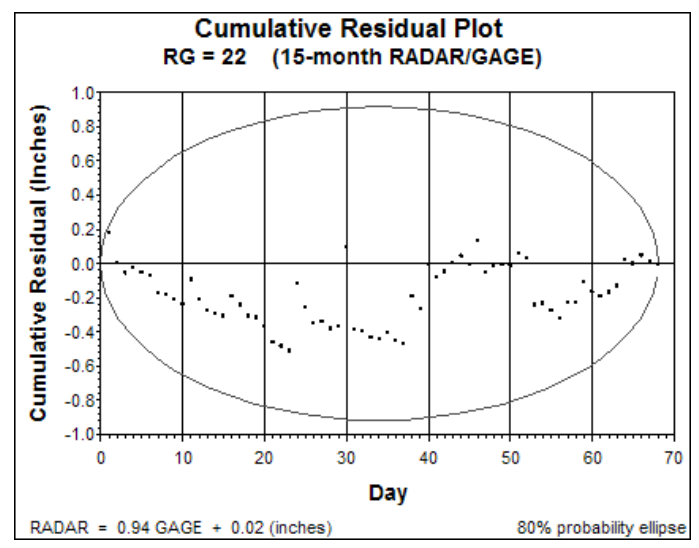

Figure 16.6 Cumulative residual time series plot from the linear regression of daily radar rainfall estimates against rain gauge totals at PWD rain gauge 22 for the 15 -month radar study period. 
Before determining the overall bias of a rain gauge record relative to the calibrated radar rainfall estimates, the homogeneity of the data sets should be verified. The verification is performed by examining the time series plots of the cumulative residual from the linear regression of daily radar against rain gauge rainfall totals, as shown for PWD rain gauge 22 in Figure 16.6. Once an acceptable degree of homogeneity between the datasets is determined for the 15-month radar study period, the spatial bias adjustment factor is calculated for the complete gauge record. The program developed for determining overall site bias factors at each gauge is a two-part process using the same techniques developed for homogenization of the gauge record.

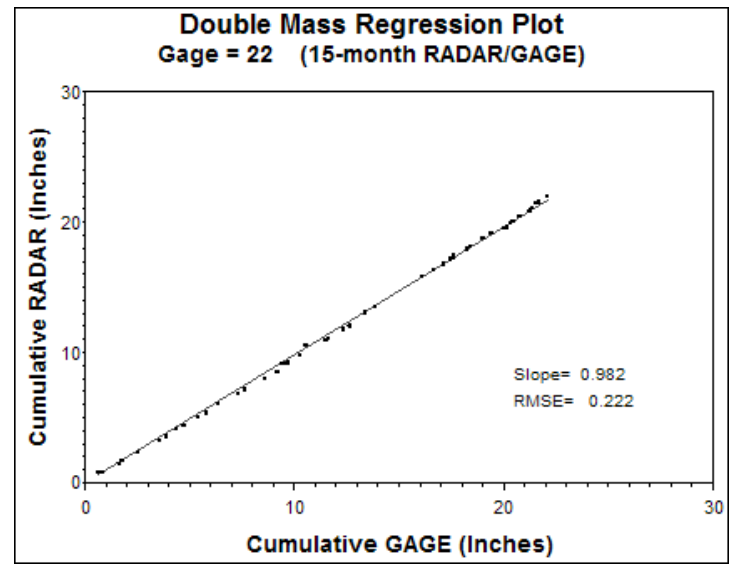

Figure 16.7 Double Mass Regression plot of cumulative daily radar against gauge rainfall totals at PWD rain gauge 22 for the 15 -month radar study period.

The first step in determining the overall spatial bias adjustment factor, once the homogeneity of all datasets is established, is to determine the bias at each gauge using the double mass regression slope,of radar to rain gauge daily rainfall totals for the 15-month radar study period as presented in Figure 16.7 for PWD rain gauge 22 .

Next, the gauge bias for the radar study period is related to the overall bias of the gauge record to determine spatial bias adjustment factors that are applied to adjust the entire period of record for the gauge, not just the 15month radar period. This is done by determining the average gauge bias for the 15-month radar period using the DMRS of the daily gauge versus the mean rainfall for this period as shown by Figure 16.8. The DMRS for the 
entire period of record was previously determined using all homogenized data as shown by Figure 16.4. Then the ratio of the DMRS for the entire period of record (all data) to that of the radar study period (15-month) is calculated. This ratio is multiplied by the DMRS radar to rain gauge bias from Figure 16.7 , to yield the overall spatial bias adjustment factor for each gauge.

\subsubsection{Results}

The overall spatial bias adjustment factors determined for each of the PWD rain gauges is presented in column 8 of Table 16.1. PWD rain gauge 14 is removed from the analysis because it lacks a sufficient number of daily measurements corresponding to the 15-month radar rainfall study period.

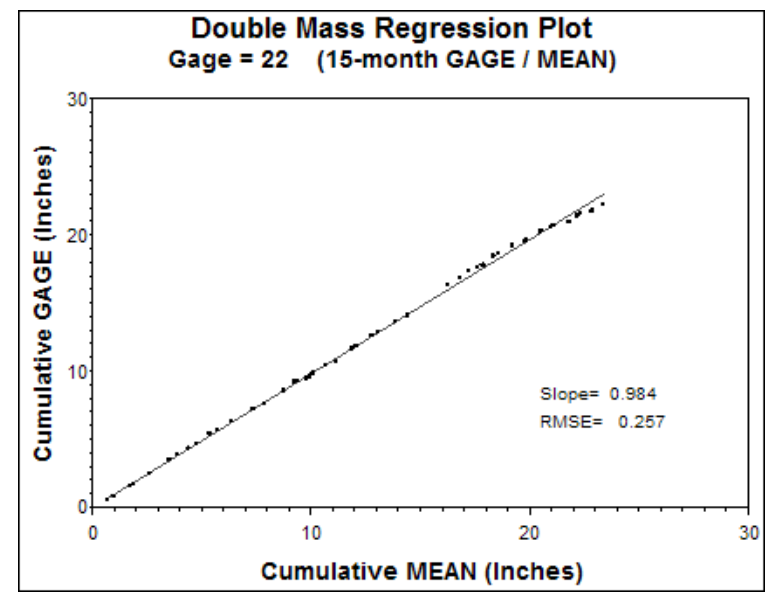

Figure 16.8 Double mass regression plot of cumulative gauge to mean daily rainfall totals at PWD rain gauge 22 for the 15 -month radar study period.

Inspection of column 3 in Table 16.1 reveals that across the network PWD rain gauges 18 and 21 exhibit the greatest overall biases in raw gauge data relative to the mean observed across the network with overall biases of negative and positive $8 \%$ respectively. These two rain gauges are located among five PWD stations covering an approximately four square mile region of North West Philadelphia. This represents an average difference in rainfall between these two gauges of approximately $15 \%$ over approximately a two mile distance. 
Table 16.1 Final Spatial Bias Adjustment. Philadelphia Water Department 24-Raingage Network Data with NEXRAIN calibrated radar mosaic

\begin{tabular}{|c|c|c|c|c|c|c|c|c|c|}
\hline 1 & 2 & 3 & 4 & 5 & $6=5 / 4$ & 7 & $8=6 \times 7$ & 9 & 10 \\
\hline \multirow{2}{*}{ 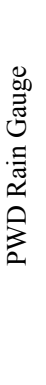 } & \multirow{2}{*}{ 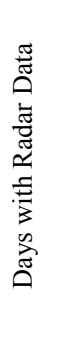 } & \multirow{2}{*}{ 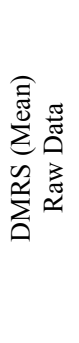 } & \multicolumn{2}{|c|}{ 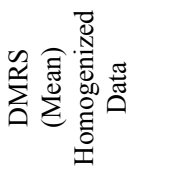 } & \multirow{2}{*}{ 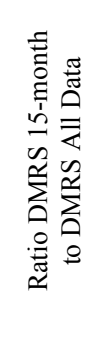 } & \multirow{2}{*}{ 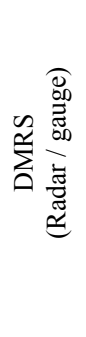 } & \multirow{2}{*}{ 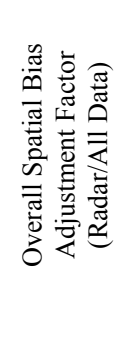 } & \multirow{2}{*}{ 兽莺 } & \multirow{2}{*}{ 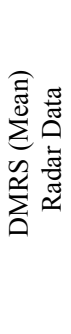 } \\
\hline & & & 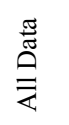 & 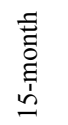 & & & & & \\
\hline 1 & 136 & 1.02 & 1.02 & 1.02 & 1.00 & 0.95 & 0.95 & 0.99 & 0.98 \\
\hline 2 & 136 & 0.99 & 0.99 & 0.95 & 0.97 & 1.01 & 0.98 & 0.99 & 0.97 \\
\hline 3 & 120 & 0.96 & 0.97 & 1.01 & 1.04 & 0.99 & 1.03 & 1.03 & 1.01 \\
\hline 4 & 46 & 0.97 & 0.98 & 0.98 & 1.00 & 0.97 & 0.97 & 0.97 & 0.96 \\
\hline 5 & 123 & 0.99 & 1.01 & 1.02 & 1.01 & 1.02 & 1.03 & 1.07 & 1.06 \\
\hline 6 & 151 & 1.02 & 1.03 & 0.99 & 0.96 & 0.98 & 0.94 & 0.99 & 0.98 \\
\hline 7 & 132 & 1.05 & 1.04 & 1.02 & 0.99 & 0.94 & 0.93 & 0.98 & 0.98 \\
\hline 8 & 75 & 0.99 & 1.01 & 0.99 & 0.98 & 1.00 & 0.98 & 1.02 & 0.98 \\
\hline 9 & 122 & 1.00 & 1.00 & 1.07 & 1.07 & 0.88 & 0.94 & 0.97 & 0.98 \\
\hline 10 & 106 & 1.02 & 1.02 & 1.02 & 0.99 & 0.95 & 0.94 & 0.99 & 0.99 \\
\hline 11 & 37 & 0.96 & 0.99 & 1.02 & 1.03 & 0.94 & 0.97 & 0.99 & 1.00 \\
\hline 12 & 41 & 0.92 & 0.97 & 0.93 & 0.96 & 1.01 & 0.98 & 0.97 & 0.98 \\
\hline 13 & 13 & 0.93 & 0.93 & 1.16 & 1.25 & 0.84 & 1.05 & 1.01 & 1.04 \\
\hline 14 & NA & NA & NA & NA & NA & NA & NA & NA & NA \\
\hline 15 & 147 & 1.03 & 1.02 & 1.01 & 0.99 & 0.99 & 0.99 & 1.03 & 1.02 \\
\hline 16 & 57 & 1.01 & 0.99 & 0.99 & 0.99 & 1.00 & 0.99 & 1.01 & 1.00 \\
\hline 17 & 144 & 1.05 & 1.04 & 1.05 & 1.01 & 0.92 & 0.93 & 0.99 & 0.97 \\
\hline 18 & 147 & 0.92 & 0.94 & 0.94 & 1.00 & 1.06 & 1.07 & 1.03 & 1.03 \\
\hline 19 & 99 & 1.00 & 1.00 & 1.02 & 1.01 & 0.95 & 0.96 & 0.99 & 0.98 \\
\hline 20 & 112 & 1.03 & 1.00 & 0.97 & 0.97 & 1.04 & 1.01 & 1.04 & 1.06 \\
\hline 21 & 70 & 1.08 & 1.04 & 0.98 & 0.95 & 0.97 & 0.92 & 0.98 & 0.97 \\
\hline 22 & 68 & 0.99 & 1.00 & 0.98 & 0.98 & 0.98 & 0.96 & 0.99 & 0.97 \\
\hline 23 & 44 & 0.95 & 0.96 & 1.02 & 1.06 & 0.88 & 0.93 & 0.91 & 0.91 \\
\hline 24 & 43 & 0.99 & 0.99 & 0.98 & 0.99 & 1.03 & 1.02 & 1.04 & 1.06 \\
\hline
\end{tabular}




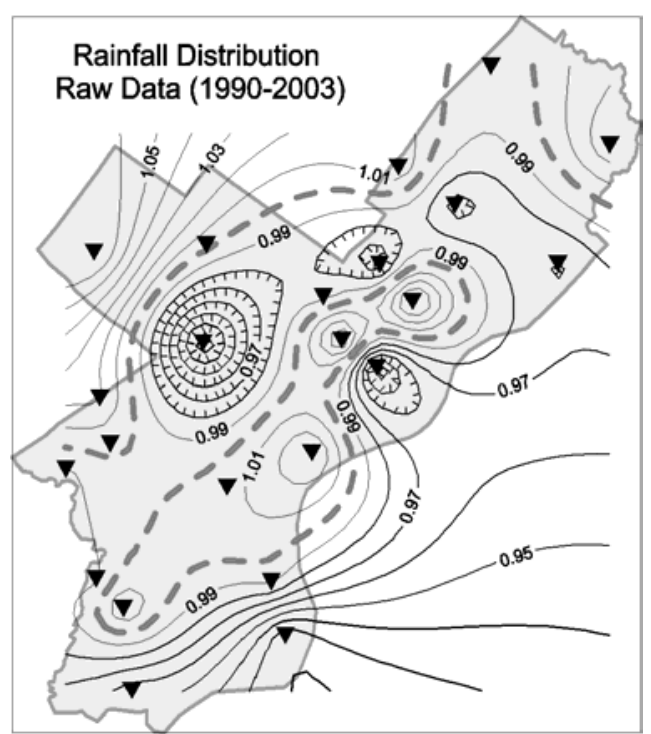

Figure 16.9 Relative rainfall distribution map of the Philadelphia area showing double mass regression slopes of PWD gauge to mean daily rainfall totals using raw data for the period 1990-2003.

The spatial distribution represented by radar rainfall data in column 10 of Table 16.1, however, reveals an average bias relative to the mean at gauges 18 and 21 of positive and negative 3\%, respectively. The spatial bias adjustment factors for PWD gauges 18 and 21 are the greatest in the network. The final results of homogenization and spatial bias adjustment, given by the gauge to mean double mass regression slopes presented in column 9 of Table 16.1, represent the long term average relative rainfall distribution across the region.

To better visualize the spatial distribution of rainfall represented by the final and intermediate results presented in Table 16.1, surface contour plots were generated using the DMRS for all rain gauge locations relative to the network mean. The long term average distribution of rainfall over the Philadelphia area illustrated in Figure 16.9 is determined from the double mass linear regression slope, column 3 of Table 16.1, using all raw data for the 1990-2003 period of record.

Isometric contours lines are generated from this data using the kriging method of spatial interpolation provided by Surfer ${ }^{\mathrm{TM}}$ for Windows Notes V6 CGolden Software Incorporated, 1993-97. 
The average rainfall distribution relative to the network mean over the 15month radar study period is presented in Figure 16.10 for raw, homogenized but not spatial bias adjusted rain gauge data. This figure reveals gauge locations with significant long and short term differences in rainfall from nearby gauges, as well as the regional average.

The final overall spatial bias adjustment factors for all homogenized PWD rain gauge data over the 1990-2003 period of record, are given in column 8 of Table 16.1 expressed as differences from the mean. These factors are presented in Figure 16.11. Note that bias adjustment factors range from plusto-minus seven percent.

The average relative distribution of rainfall observed from the radar rainfall data over the 15-month study period (Figure 16.12) compares favorably to that of the final adjusted PWD rain gauge data determined over the 1990-2003 period of record (Figure 16.13).

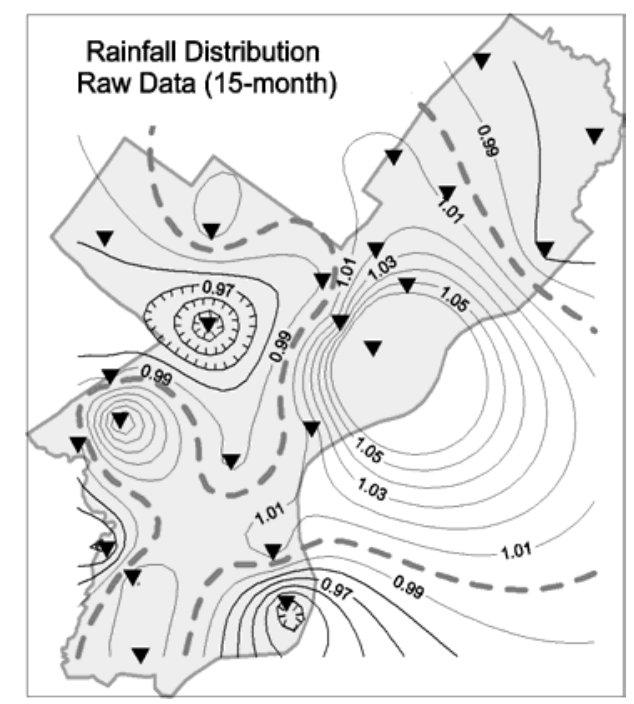

Figure 16.10 Relative rainfall distribution map of the Philadelphia area showing double mass regression slopes of PWD gauge to mean daily rainfall totals for the 15-month radar study period. 


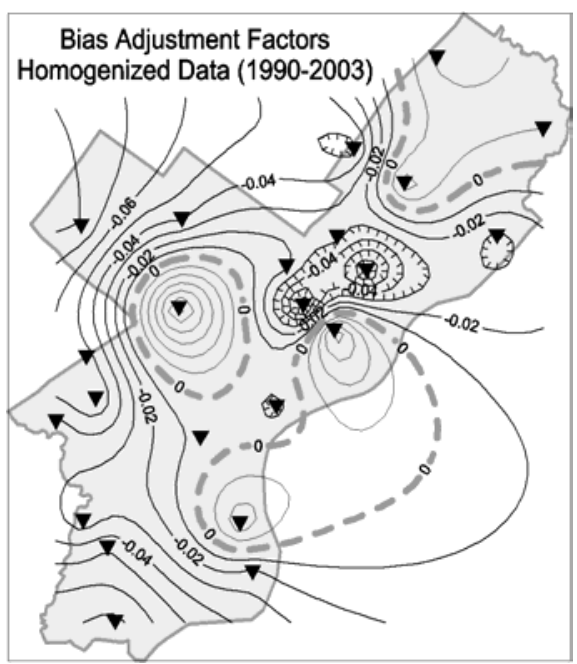

Figure 16.11 Overall spatial bias adjustment factor map using homogenized PWD rain gauge data for the period 1990-2003.

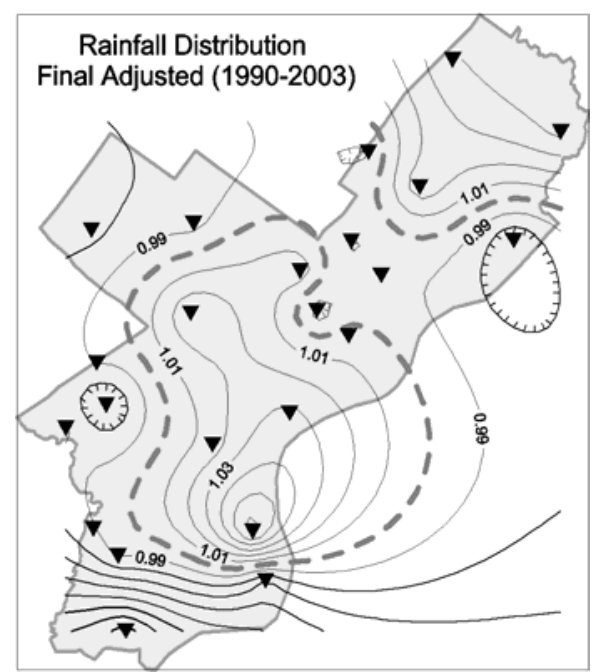

Figure 16.12 Rainfall distribution map of the Philadelphia area using radar data over the 15-month study period. Contours are double mass regression slopes at gauge locations relative to the mean.

Martens and Smullen: Normalizing Rain Gauge Network Biases with Calibrated Radar Rainfall Estimates. In: Effective Modeling of Urban Water Systems, Monograph 13. James, Irvine, McBean \& Pitt, Eds. ISBN 0-9736716-0-2 @ CHI 2005 www.computationalhydraulics.com 


\subsection{Evaluating Adjusted Rain gauge Data Using Hydrologic Model Calibration Results}

Evaluating and adjusting the homogeneity and relative gauge biases across a rain gauge network controls uncertainty in deviations in predicted versus monitored flows. The effectiveness of bias normalization in reducing model uncertainty is evaluated by comparing the results of model simulations using both adjusted and non-adjusted rainfall datasets, and can be measured by the root mean square error (RMSE) of predicted versus observed event volumes. The magnitude of the improvement varies depending on the importance that gauges with highly adjusted data sets play in the system model.

\subsubsection{Dataset}

Hydraulic model results are compared to flow monitoring data for the PWD combined trunk sewer temporary flow monitoring site, TC06. The ninetyseven-acre drainage basin for site TC06, located in the region north of PWD rain gauge 22, is modeled as part of the City of Philadelphia's South West Water Pollution Control Plant combined sewer system model.

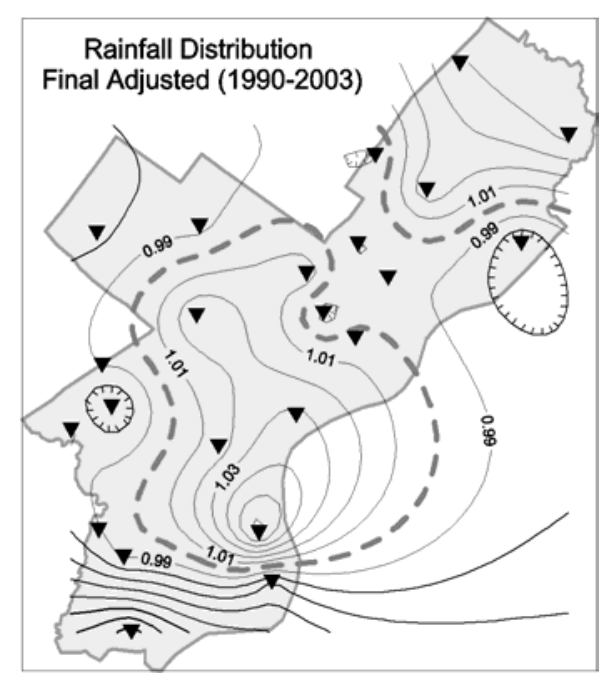

Figure 16.13 Rainfall distribution map using final adjusted PWD rain gauge data for 1990-2003. Contours from double mass regression slopes at gauge locations relative to the mean. 
Runoff is generated from this basin with input rainfall time series data from PWD rain gauge 22. Twelve month continuous SWMM RUNOFF and EXTRAN simulations were performed for the flow monitoring period October 2002 through November 2003 using both adjusted and non-adjusted rainfall data for comparison.

\subsubsection{Homogeneity of Flow Monitoring Data and Model Calibration Event Selection}

Cumulative residual time series analysis techniques used to evaluate the homogeneity of rain gauge data also can be employed as part of the quality assurance and quality control procedures to evaluate the homogeneity of flow monitoring data and selecting monitored storms for model calibration. Inspection of the cumulative residual model versus monitor time series plots benefits identification of potential changes in the modeled verses monitored relationship over time. If a significant change in the homogeneity of this relationship is observed over the calibration monitoring period, it must be determined if this change represents non-homogeneity of precipitation and flow monitoring time series, or a physically based change in the rainfallrunoff relationship.

The cumulative residual plot of model versus monitor event volumes for forty-five monitored storm events for site TC06 are presented in Figure 16.14. A significant change in the model to monitor relationship can be readily seen from this plot to occur after approximately event 34, corresponding to August 5,2003 . A similar change in the homogeneity of the rain gauge data is not observed in the cumulative residual plot of gauge to mean daily rainfall totals presented for PWD rain gauge 22 in Figure 16.15. The change in the model to monitor relationship observed in Figure 16.14 therefore represents either a change in the flow monitoring conditions or a physically based, perhaps seasonal, change in the rainfall-runoff relationship. Further analysis of the flow monitoring data using hydrograph separation techniques reveals an increase in the frequency of events, beginning in August of 2002, that exhibit significant decreases in base flow rates. This indicates a change in the flow monitoring conditions that may render events unsuitable for calibration analysis. Eliminating events from the analysis that exhibit a significant decrease in base flow rates over the event duration leaves twenty-two events for the calibration analysis. The cumulative residual time series plots of model to monitor event volumes for the twenty-two events selected for final 
calibration analysis at site TC06 is presented in Figure 16.16. In addition, the cumulative residual plot of gauge to mean daily rainfall totals is presented for these events in Figure 16.17, verifying that the dataset used at site TC06 is homogeneous over the calibration period.

\subsubsection{Effect of Rainfall Adjustment on Model Calibration Results}

The effect of rainfall data adjustment on model calibration results can now be evaluated using the twenty-two stormwater flow events monitored at site TC06. Scatter plots of model versus monitor event volumes at PWD combined sewer monitoring site TC06 are presented in Figures 16.18 and 16.19. Comparison of results obtained before and after bias adjustment of rain gauge 22 , reveals an over $20 \%$ reduction in the root mean square error, RMSE, of predicted versus observed event volumes at site TC06. While individual event volumes are predicted closer to the observed values before bias adjustment of rainfall data, on average, the ability of the model to predict event volumes is improved by bias adjustment.

As noted previously, the double mass regression slope, compared to the simple regression slopes presented in Figures 16.18 and 16.19, is a more stable measure in terms of the influence of large event outliers for characterizing the average relationship between two time series. The average relationship between predicted and observed event volumes at site TC06 changes significantly before and after bias adjustment as seen from the double mass regression plots of model verse monitor volumes presented in Figures 16.20 and 16.21 .

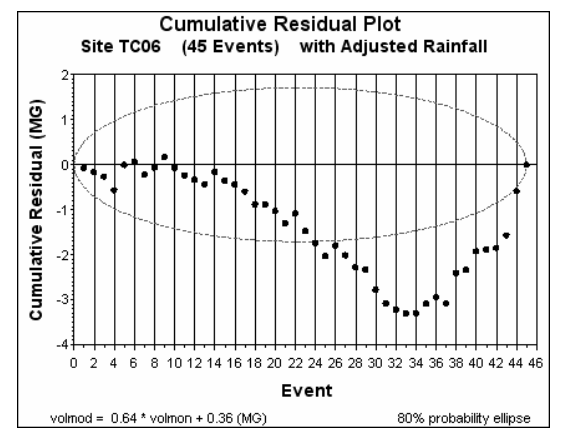

Figure 16.14 Cumulative residual time series plot of model versus monitor event volumes at PWD site TC06 for forty-five storm events using adjusted rainfall data from PWD rain gauge 22 . 


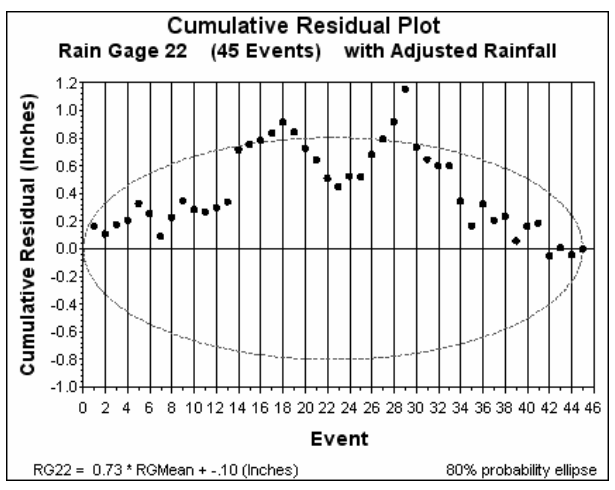

Figure 16.15 Cumulative residual time series plot of gauge versus mean daily rainfall totals for forty-five storm events using adjusted rainfall data from PWD rain gauge 22.

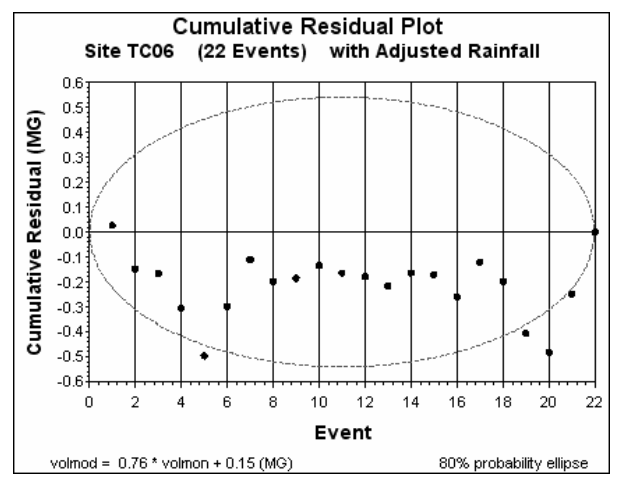

Figure 16.16 Cumulative residual time series plot of gauge versus mean rainfall totals for twenty-two storm events using adjusted rainfall data from PWD rain gauge 22.

This is a $24 \%$ reduction in model event volumes when using adjusted compared to non-adjusted rain gauge data.

This significant reduction in the model estimation of total event volumes is the result of the homogeneity adjustment of rain gauge data for PWD rain gauge 22 presented in Section 2. A significant change in rain gauge bias is observed in Figure 16.3 beginning approximately at day 1100, corresponding to August 20, 2002. 


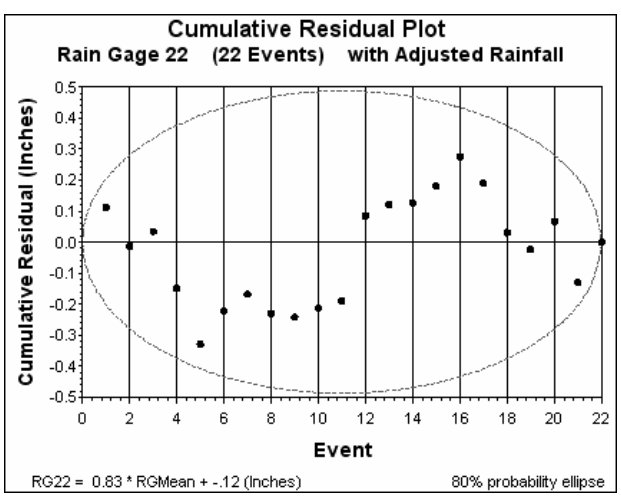

Figure 16.17 Cumulative residual time series plot of model versus monitor event volumes at PWD site TC06 for twenty-two storm events using adjusted rainfall data from PWD rain gauge 22 .

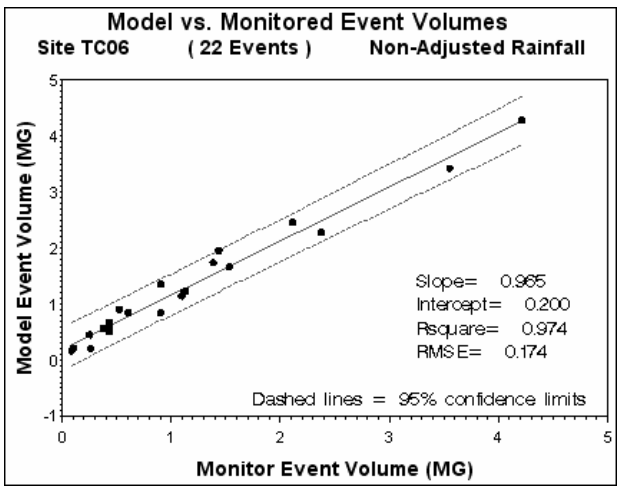

Figure 16.18 Scatter plot of model versus monitor event volumes at PWD site TC06 using non-adjusted rainfall data from PWD rain gauge 22.

This is corrected by the homogeneity adjustment factor applied for the period which uniformly reduces the rainfall reported at the gauge.

\subsection{Conclusions and Recommendations}

Most long term precipitation time series contain significant nonhomogeneities with respect to the actual precipitation occurring at the site over time (Peterson et al 1998). Furthermore, few station records can be considered homogeneous relative to data from surrounding gauges (Easterling 
et al 1995). Non-climatic changes in precipitation measurements can commonly range from $4 \%-40 \%$ within a few years (Dia et al 1996).

Evaluating the homogeneity of precipitation time series is important whenever using this data for model calibration, long term continuous simulations, or frequency analyses. It is important whether creating a single homogeneous precipitation time series or multiple time series representing spatial variation across a study area.

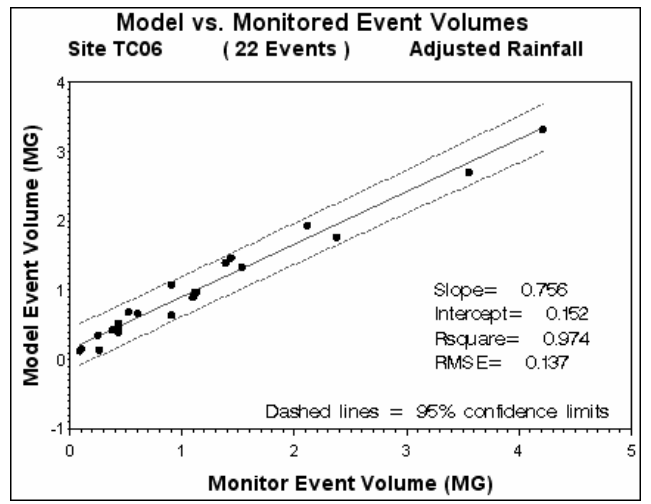

Figure 16.19 Scatter plot of model versus monitor event volumes at PWD site TC06 using adjusted rainfall data from PWD rain gauge 22.

These techniques depend on the homogeneity of a highly correlated reference time series and can be limited by the availability of sufficient spatial or temporal database. The proportional effect that homogeneity and spatial bias adjustment of rain gauge data has on hydrologic model calibration results is shown to be significant for the monitoring period investigated at PWD site TC06 as seen by a nearly $25 \%$ reduction in the average ratio of model to monitor event volume. The importance of evaluating and adjusting homogeneity and relative gauge biases across a rain gauge network clearly is demonstrated.

Improvement in the correlation of modeled to monitored stormwater runoff volumes for a twelve-month period at PWD flow monitoring site TC06 is evidenced by a nearly $20 \%$ reduction in the root mean square error after rain gauge data adjustment. 


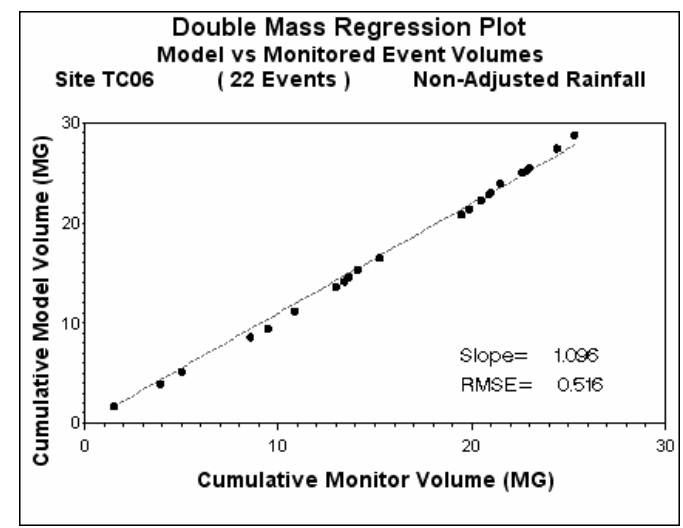

Figure 16.20 Double mass regression plot of cumulative model versus monitor event volumes at PWD site TC06 before bias adjustment of data from PWD rain gauge 22.

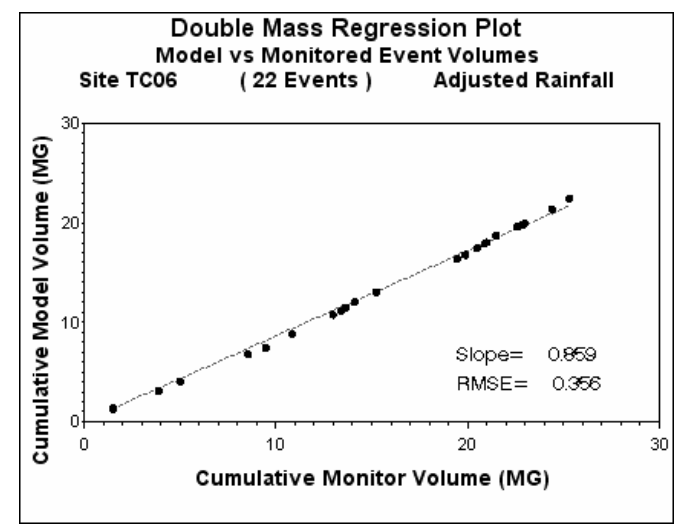

Figure 16.21 Double mass regression plot of cumulative model versus monitor event volumes at PWD site TC06 using adjusted rainfall data from PWD rain gauge 22.

The additional confidence gained in our knowledge of the rainfall runoff relationship through the use of cumulative residual time series analysis techniques has proven valuable for the quality assurance and quality control of rain gauge as well as flow monitoring data used for long term continuous hydrologic model calibrations. 


\subsubsection{Recommendations}

Investigations designed to measure improvements in model to monitor correlations resulting from the use of homogeneity and spatial bias adjusted rain gauge data should further incorporate long term continuous simulation results at numerous flow monitoring locations across the study basin.

The sensitivity of cumulative residual time series analysis techniques to reveal changes in the model to monitor relationship suggests further investigations to identify and calibrate the modeling of seasonal or other changes observed in the rainfall runoff relationship over time.

Application of objective statistical methods for use in defining the limits of homogeneous adjustment periods may be a useful addition to the rain gauge network bias normalization procedure presented in this study.

\section{References}

Allen, Richard G. and Pereira, Luiss with contributions by Teixeira J.L., 1998: Crop evapotranspiration - Guidelines for computing crop water requirements - FAO irrigation and drainage paper 56. Food and Agriculture Organization of the United Nations, Rome, Annex 4. Statistical analysis of weather data sets.

Alexandersson, Hans, 1986: A homogeneity test applied to precipitation data. J. Climate, 6: 661-675.

Craddock, J.M. 1979: Methods of comparing annual rainfall records for climate purposes. Weather 34: 332-345.

Dai, Aiguo, Fung, Inez Y. and Del Genio, Anthony D., 1997: Surface observed global land precipitation variations during 1900-88, J. Climate, 10: 2943-2962.

Easterling, David R. Peterson, Thomas C. and Karl Thomas R., 1995: Notes and correspondence: On the development and use of homogenized climate datasets. J. Climate, 10: 1429-1434.

Guttman, Nathaniel B., 1998: Hogeneity, Data Adjustments and Climatic Normals, National Climatic Data Center, 151 Patton Avenue, Ashville, NC 28801-5001.

Henriques, Raquel and Santos, Maria Joao, 1999: Analysis of the European annual precipitation series, Technical report to the ARIDE project No.3: Water Institute, DSRH - Av. Alimrante Gago Coutinho 30, 1049-066 Lisbon, Portugal

Peterson, T.C., Easterling, David R., Karl, Thomas R., Groisman, Pavel, Nicholls, Neville, Plummer, Neil, Torok, Simon, Auer, Ingeborg, Boehm, Reinhard,Gullett, Donald, Vincent, Lucie, Heino, Raino, Tuomenvirta, Heikki, Mestre, Olivier, Alexandersson, Hans, Jones, Philip and Parker, David, 1998: Homogeneity adjustments of in situ atmospheric climate data: A review. Intl. J. Climatol. 18: 1493-1517.

Vincent, Lucie A., 1998: Techniques for the identification of inhomogeneities in Canadian temperature series. J. Climate, 11: 1094-1104. 